\title{
Daylight Simulation base on real daylight LED module
}

Abstract. Many lighting companies and light source producers offer daylight simulation on base of different CCT changins. More or less this has nothing to do with real daylight which has completely different spectrum. Our research is focused on complete spectrum of a light source which is able to replace dalyight. As first step we selected reference standard D50. Spectrum of light source matched in $95 \%$ with spectrum of a real daylight with CCT 5000 K. Application for this kind of light source can be in workplace where people works for 3 shifts. Under this conditions is important that human internal clock works optimal as under the normal daylight.

Keywords: daylight, LED, spectrum.

\section{Introduction}

It has long been known that the natural light of the sun is beneficial for our well-being. Simply notice the bounce in people's steps and the smiles on their faces when the sun finally peeks out from behind the clouds after an extended absence in the midst of a dreary, cold winter. Most people are fully aware of the impact sunlight can have on emotional well-being. It's difficult to envision a setting with limited sunlight and smiling inhabitants. Aside from the emotional benefits, daylight has been shown to have a significant impact on productivity and health. Our eyes and brains simply function more optimally with natural light versus artificial lighting. It has also been observed, especially in comparisons of schools with sufficient daylight over schools reliant on artificial lighting, that children are able to concentrate and maintain focus better when there is ample natural light. It is not surprising that these observations also signify the benefits of natural light on health and improved energy levels. Regardless of the sophistication of technology, humans are natural beings in need of natural energy. The sun is our energy source and we are in need of its energy daily in order to function optimally.

Many scientists agree with ordinary people that daylight is ideal light source. There are plausible reasons for this statement, for example:

- Daylight/sunlight is the most common light source available and it is here for million years therefore human vision has been adapted especially for this light source.

- Spectral content of the daylight is smooth and rich for each color hue.

Color appearances of objects have tendencies to be more attractive during bright days and these are the colors we like to remember and unconsciously compare with.

\section{Research}

Our daily experience with the daylight has taught us that spectral power distribution of the daylight is influenced by many factors like: (i) cloud cover, (ii) aerosol content - fog or smog, (iii) hour and season and also (iv) geographic orientation and location. However, human eye can adapt for each set of conditions and perform very well. These are good qualities and CIE (International Commission for Illumination) has selected daylight as one of reference light sources, in particular reference sources D. For this purpose the daylight has to be unambiguously quantified which has been achieved by means of analyzing thousands of measurements. CIE couples spectral power distributions of the $\mathrm{D}$ sources with correlated color temperature, CCT, of
Planckian radiators. Details can be found e.g. in CIE 15:2004 Colorimetry.

The origin of the daylight is ultimately Sun, which is in fact a big hot sphere. The surface temperature is over 5000 degrees Celsius. Its emitted radiation follows Planck's law for black body with perturbations in the spectra due to absorption present in Sun and atmosphere of Earth and the reasons mentioned above. This can be clearly observed in Figure 1 and 2 where two the most common reference daylight spectra D50 (characteristic for morning daylight) and D65 (characteristic for noon daylight) are shown together with smooth Planckian spectra of their corresponding black body radiators.

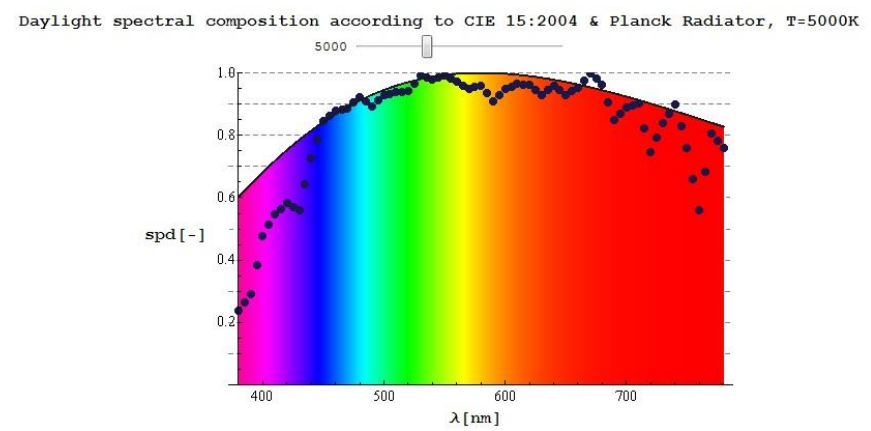

Figure 1: Spectral Power Distribution of Planckian radiator having $\mathrm{T}=5000 \mathrm{~K}$ and daylight D50.

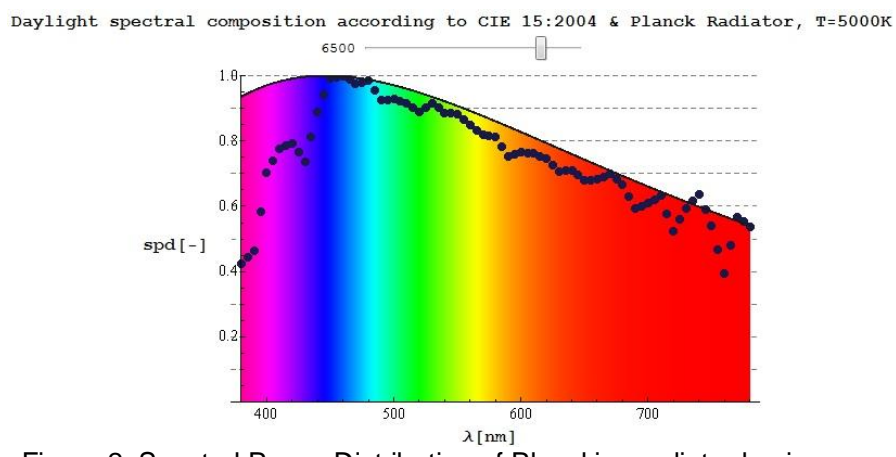

Figure 2: Spectral Power Distribution of Planckian radiator having $\mathrm{T}=6500 \mathrm{~K}$ and daylight D65.

Now we have the knowledge what spectral shape of light sources is needed in order to get natural daylight into our offices and corridors from luminaires. How to achieve that? A straightforward way is to mimic the nature, thus to get a really hot object with temperature above $5000 \mathrm{~K}$ and appropriate absorption. Nowadays state-of-the-art technology is not advanced enough to build a reliable "heater" and its housing. Even if it was possible it would definitely not be efficient! Nonetheless LEDs in conjunction 
with phosphor/luminophore technology are able to offer economic and efficient solutions which details are given below. We note that spectral range from 450 to $700 \mathrm{~nm}$ is going to be matched at this stage.

Generation of blue or violet light has become very efficient and being further increased rapidly year by year. Phosphor/luminophore technology has been improving simultaneously and can offer wide range of efficient, stable phosphors in terms of peak wavelength and bandwidth of the emitted (converted) light. Combination of commercially available appropriate phosphors pumped by violet or blue LEDs and commercially available colored LEDs can already produce interesting spectra. Simulation was performed, which results are shown in Figure 3 and 4, using both measured spectra (Luxeon Rebel, pumped phosphor) and appropriate models of LED/phosphor emission spectra (Luxeon Rebel). At the top of the both Figures there are sliders, which control LEDs' amplitude and peak wavelength and bandwidth for the modelled spectra. The below section of the Figures is made of two graphs.

The left graphs show:

1. Reference daylight D50 which is made of discrete points, filled by rainbow colors and that shall be matched.

2. Resulted spectrum (solid line) obtained by combining emitted light from the phosphors and colored LEDs, in this case four colors.

The right graphs show spectra of the light emitted by the pumped phosphor filled by rainbow color.

Figure 3 shows the resulting spectrum which $\mathrm{CCT}=5000 \mathrm{~K}$. The spectrum is more perturbed at the blue spectral region due to the large gap between the pump and emission spectrum (right graph) of the phosphor. In fact all the colored LEDs bridge the gap and the result is not smooth.

Figure 4 shows the spectrum of the 4 LEDs (royal blue, blue, cyan, lime) which were used in order to obtain good spectral match (thus the right graph is zero).

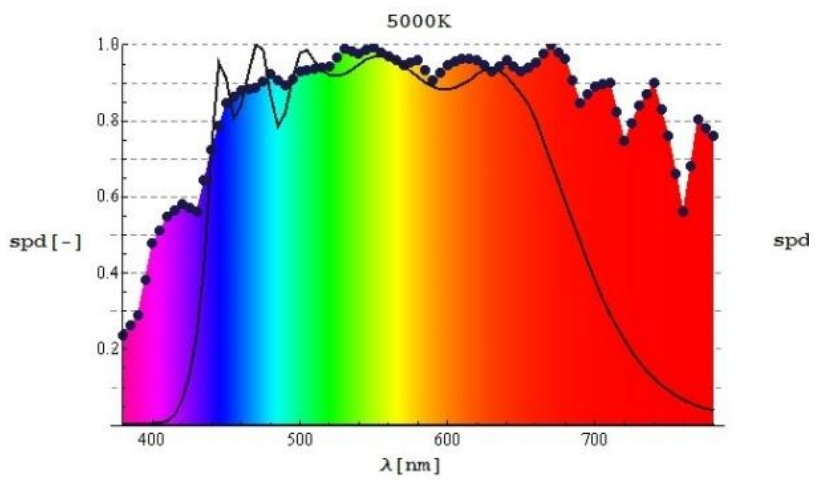

Figure 3: combination of the emission spectrum of the phosphor and colored LEDs.

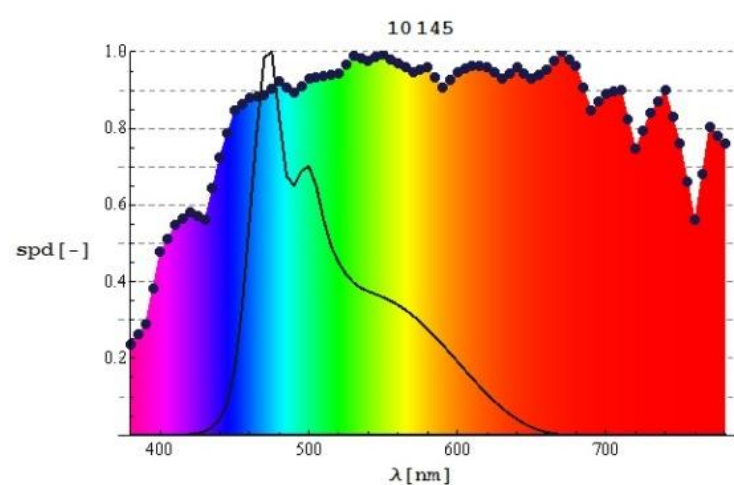

Figure 4: emission spectra of the colored LEDs.

Predictions of the above simulation will be verified experimentally in coming days. Prototype of a light source is being constructed which will employ two mixing chambers and five colored LEDs. There is a potential for even better and/or broader spectral match.

\section{Prototype}

Base on research which was described above we were evaluation different ways of prototyping how to bring our research to the usable piece of LED module which can be used in luminaires which are responsible for general lighting. LED module should be solid enough for later test processes which we do in our laboratories. Selection of materials which will be used were discussed with producers to find best material to do a job which is requested.

\section{Mixing chamber}

As a base for mixing chamber was selected 3D printing method. Design of mixing chamber correspond with recommendations and design guide from producer of "remote phosphor" parts. Mixing chamber has to be done in that way that only limited part of the emitted blue light from "blue pumps" can be absorbed or transmitted via mixing chamber walls. This is the most important factor which can increase or decrease efficiency of the LED module. Important is surface treatment which has to be nonselective (treatment does not change spectral distribution of reflected light). Another 'part of mixing chamber is a cover layer made of MCPET material which has reflectance $98 \%$. It has to be also non-selective material. This part is important also from efficiency point of view. In our tests we found out that difference can be up to $15 \%$ with or without this part. Mixing chamber has middle part which separates coloured LEDs from Blue pumps. Treatment of internal walls of mixing chamber was done base on barium sulphide for the best reflectivity properties. Many producers of integrating spheres are using similar surface treatment for internal reflective parts of integration spheres.

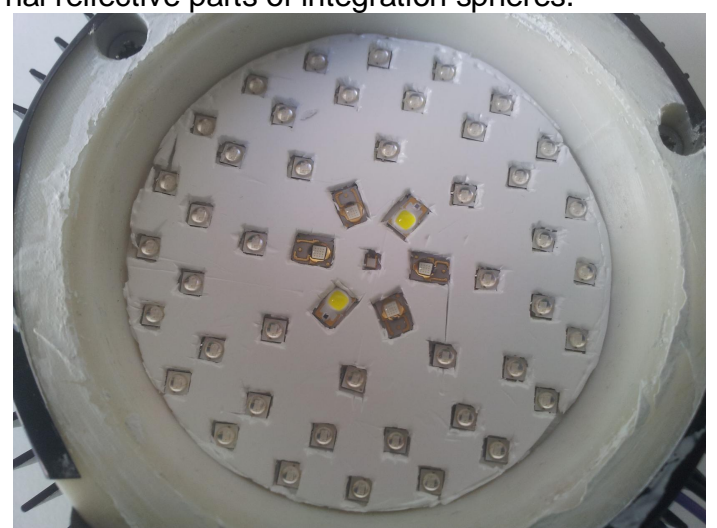

Fig. 5 Detail of printed mixing chamber with cover plate. 


\section{PCB design}

PCB design was done in our research centre base on previous calculation of different coloured LEDs and blue pumps with remote phosphor. PCB for D50 simulator was designed in that way that each part is controlled separately. Blue pumps and coloured LEDs works separately and independently. It means that we control blue pumps for overall spectrum and each coloured LED to tune up the spectrum. The goal of this project is to have only one driver which is able to supply D50 simulator and "smart PCB" which will split voltage and current for LEDs exactly how it should be. Precision of this has to be on highest level because each difference can cause in spectral differences which will change final spectrum of LED module. We know that there are different bins of LEDs so we need to calibrate each module independently according to spectral emission.

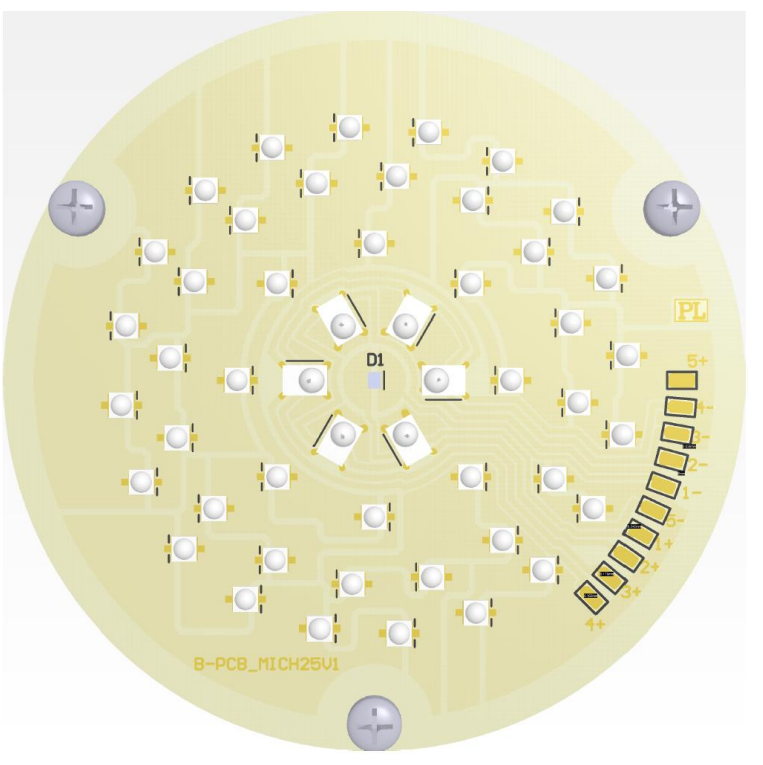

Fig. 6 PCB+LEDs.

\section{Diffusers}

Selection of diffusers was split to the two different parts. First was selection of diffuser with remote phosphor technology to convert blue light from blue pumps to the white light with needed spectrum. This part was done in cooperation with Intematix (producer of remote phosphors). Remote phosphor has to be hand in hand with blue pumps to get most from it. Biggest part of resulted spectrum is done by this combination. It has CRI 90 and efficiency up to $110 \mathrm{~lm} / \mathrm{W}$. There is so many advantages to use a remote phosphor. Colour stability over the lifetime, high CRI, spectral emittance, etc.

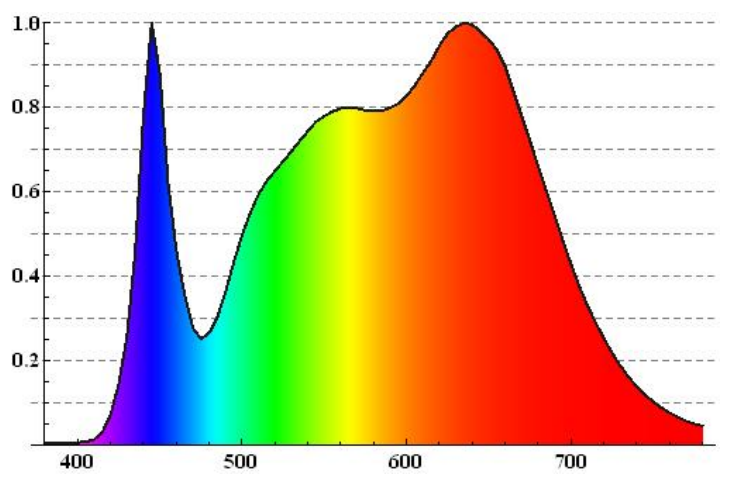

Fig. 7 Emission spectrum of remote phosphor and blue pumps.

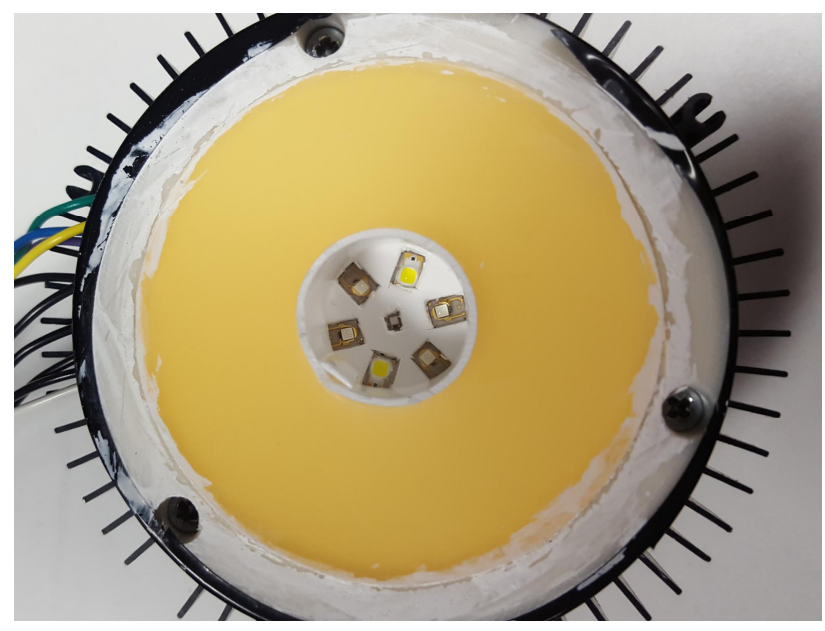

Fig. 8 Detail of remote phosphor and coloured LED.

\section{Measurement}

Measurement of D50 simulator and resulted spectrum was done in integrating sphere from LabSphere (Diameter $2 \mathrm{~m}$ )(fig.9). Each part of PCB has to be set up with correct voltage and current. After calibration and tuning up the module we did several spectral measurements. Basic set up of devices is shown in fig. 10.

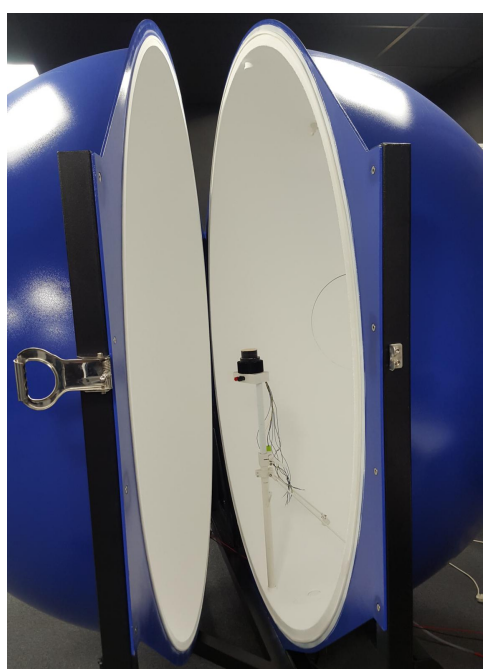

Fig. 9 D50 simulator in integrating sphere.

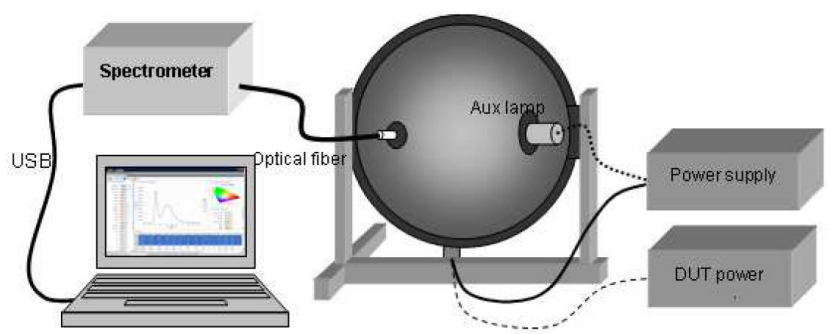

Fig. 10 Basic set up of measurement devices and power supply units.

\section{Results}

D50 simulator achieved spectrum according to our calculations with high precision. We reached CRI more and CCT 5050K (tab.1) with luminous flux $1520 \mathrm{~lm} \pm 5 \%$. Power consumption is $21 \mathrm{~W}$ and efficiency $72 \mathrm{Im} / \mathrm{W}$. Final spectral measurement and chromaticity diagram are shown in fig. 11. D50 Simulator has value of R9 96 which is in case of standard LEDs the most critical point. Efficiency of such a 
module is not comparable with standard white LEDs which are available on the market. But if we think about a ful spectrum light source which is able to simulate daylight with $5000 \mathrm{~K}$ and high $\mathrm{CRI}$ it is way how we can get sunlight to each room and help to the people during their working time.

\begin{tabular}{|l|l|l|l|l|l|l|l|}
\hline \multicolumn{7}{|c|}{ D50 Simulator CCT=5050K } \\
\hline Ra & 95 & R4 & 93 & R8 & 94 & R12 & 87 \\
\hline R1 & 98 & R5 & 97 & R9 & 96 & R13 & 96 \\
\hline R2 & 96 & R6 & 96 & R10 & 92 & R14 & 97 \\
\hline R3 & 95 & R7 & 92 & R11 & 96 & R15 & 95 \\
\hline
\end{tabular}

Tab.1 Measured values for extended CRI Table

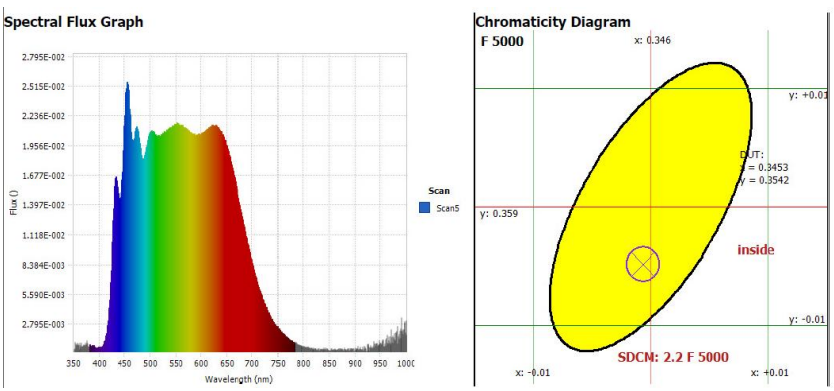

Fig.11 Spectral Flux Graph and chromaticity Diagram of D50.

Acknowledgement. This work was supported under project APVV-0118-12.

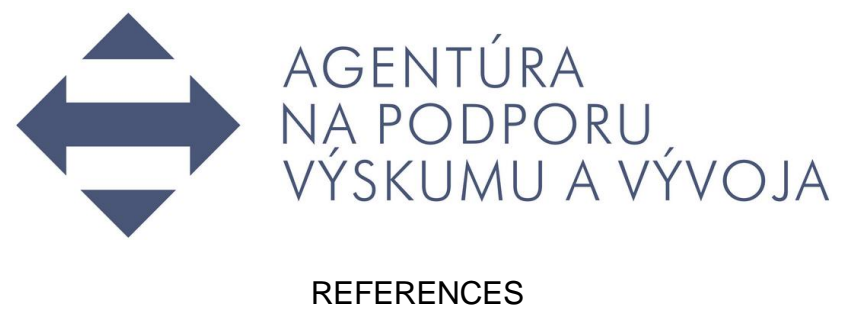

[1] A report of IEA SHC Task 21/ ECBCS Annex 29, July 2000, Daylight in Buildings, a sourcebook on daylighting systems and components

[2] http://www.intematix.com/products/chromalit

[3] G. C. Brainard, J. P. Hanifin, J. M. Greeson, B. Byrne, G. Glickman, E. Gerner, M. D. Rollag, Action spectrum for melatonin regulation in humans: evidence for a novel circadian photoreceptor, J. Neurosci. 21, pp. 6405-6412,

2001.

[4] D. Gall, Die Messung circadianer Strahlungsgrößen, 2004.

http://www.tu-

IImenau.de/fakmb/fileadmin/template/fglt/publikationen/2004/Vortra g_Gall2004.pdf Techn. Univ. IImenau.

\section{Authors:}

Ing. Marek Mácha, iLumTech. Dojč 419, 90602 Slovakia, e-mail: marek.macha@ilumtech.eu

Ing. Dušan Sabol, Ips, email: dusan.sabol@lps.sk 